\title{
Modeling and Optimization of Advanced Multilayered Absorbers
}

\author{
Ignace Bogaert ${ }^{\# 1}$, Femke Olyslager ${ }^{\# 2}$, Yoeri Ariën ${ }^{* 3}$, Davy Pissoort ${ }^{\# 4}$ \\ \# Department of Information Technology, Ghent University \\ Sint-Pietersnieuwstraat 41, 9000 Ghent, Belgium \\ ${ }^{1}$ ignace.bogaertaintec.ugent.be \\ ${ }^{2}$ femke.olyslageraintec.ugent.be \\ ${ }^{4}$ davy.pissoortaintec.ugent.be \\ *Emerson \& Cuming Microwave Products \\ Nijverheidsstraat 7A, 2260 Westerlo, Belgium \\ ${ }^{2}$ yarien@eu.eccosorb.com
}

\begin{abstract}
In this contribution we present a design environment for the optimization of layered absorbers. The manufacturer has a wide set of materials with known properties available. The thickness of these materials can vary within given limits. For a given number of layers and given absorption requirements as a function of angle of incidence, polarization and frequency we try to optimize for the thicknesses of the layers and for the best possible choice of available material types. The optimization for the layer thicknesses is done using a simulated annealing technique and for selecting materials usually the whole discrete design space is sampled. The paper is illustrated with two realizations comprising comparisons between simulations and measurements.
\end{abstract}

\section{INTRODUCTION}

High quality electromagnetic absorbers require the use of advanced layered stacks of basis materials. Manufacturers usually have a wide portfolio of available absorbing materials, some of which with variable thickness. From a concurrential point of view it is very important to be able to deliver the best possible design starting from the portfolio (or part of it) within given constraints. The constraints usually can consist of a gabarit over frequency and/or angle of incidence and specifications with respect to polarization.

The problem that we consider in this paper tries to answer the question for finding a best possible design. We assume that the number of layers is given and that the thickness of each layer needs to be optimized as well that each layer needs to be chosen from a given material portfolio. This means an optimization process in two spaces: one continuous space for the thickness of each layer and one in a discrete space to select the material from a portfolio.

Since we really want to find the best possible solution in the discrete portfolio space we opted for searching that entire space. This does not mean that always the entire portfolio of the manufacturer is tried for each layer. Usually this will be only a selected part of that portfolio because there could be other constraints that limits the set of possible materials such as weight, heat resistancy, water resistancy, cost, strength, ... . Obviously not all materials can be glued together leading to further restrictions. Nevertheless the number of points in the discrete space that need to be analyzed can be massive.

Optimization of layered media has been the subject of considerable research in the past. We refer to [1] and references therein as an entrance to literature. In [1] a genetic algorithm is proposed for the optimization. The optimization in [1] looks for the so-called Pareto front that is defined as the set of designs which are thinnest for every achievable level of reflectivity. Also the Minimax optimization algorithm has been widely used for microwave designs [2]. We however opted to use a "Simulated Annealing" approach [3]. Just as the genetic algorithm approach the simulated annealing approach looks for a global optimum. Finding an optimum for a given set of materials, i.e. for a given point in the discrete space, will require many evaluations of the reflectivity of a set of layered media.

The calculation of the reflectivity of a set of layers is done using the transfermatrix method. This allows for a very fast evaluation of the reflection coefficient. The transfermatrix method has the disadvantage of being not always numerically stable when evanescent incident waves occur somewhere in the stack of layers or when materials are lossy. A solution to this problem is the usage of a recursive transfermatrix technique as explained in [4] and [5]. The recursive transfermatrix method has the disadvantage of being considerable more time consuming than the transfermatrix method. Since we need to evaluate an enormous amount of reflectivities we opted for the transfermatrix method.

Absorbing materials usually have a strong frequency dependence. This dependence is available from measurements. This poses another problem because the measurements will contain noise and are performed with different measurement techniques over different frequency ranges. Such non-smooth curves could pose problems in the optimization. We developed an advanced scheme for smoothing the data, this however falls outside the scope of this contribution.

Although most problems are reflectivity problems one is also confronted with transmission problems. This will e.g. be the case in shielding problems or in radome design. A 
shielding problem requires the reduction of the transmission coefficient and a radome design requires the maximallization of the transmission. Both are delt with in a similar way as the reflectivity problem outlined above.

In the next section some more details are given concerning the used algorithms and in the third section we present measurement and simulation results for two practical realizations.

\section{Algorithms}

The transfermatrix method consists of a concatenation procedure of transfermatrices of layers and layer interfaces. Let us assume a TE-polarization, in which case one transfers the components of left and right propagating electric fields. The TM case is dual. The transfermatrix for an interface between layer I and II is given by

$$
\left[\begin{array}{l}
E_{I}^{+} \\
E_{I}^{-}
\end{array}\right]=\frac{Z_{I}}{2 \cos \theta_{I}}\left[\begin{array}{ll}
\frac{\cos \theta_{I}}{Z_{I}}+\frac{\cos \theta_{I I}}{Z_{I I}} & \frac{\cos \theta_{I}}{Z_{I}}-\frac{\cos \theta_{I I}}{Z_{I I}} \\
\frac{\cos \theta_{I}}{Z_{I}}-\frac{\cos \theta_{I I}}{Z_{I I}} & \frac{\cos \theta_{I}}{Z_{I}}+\frac{\cos \theta_{I I}}{Z_{I I}}
\end{array}\right]\left[\begin{array}{l}
E_{I I}^{+} \\
E_{I I}^{-}
\end{array}\right]
$$

where $Z_{I}=\sqrt{\frac{\mu_{I}}{\epsilon_{I}}}$ and $Z_{I I}=\sqrt{\frac{\mu_{I I}}{\epsilon_{I I}}}$ are the impedances of the two media adjacent to the interface and where $\theta_{I}$ and $\theta_{I I}$ are the angles of incidence in the two layers. The plus and minus sign indicate left and right propagating waves.

When designing layered absorbers often resistive sheets are used. These can be thought of as a thin layer with conductivity $\sigma$ and thickness $d$. When we simultaneously take the limit $d \rightarrow 0$ and $\sigma \rightarrow+\infty$, while keeping the product $\sigma d$ constant, a resistive sheet is formed. The transfermatrix for such a sheet is given by

$$
\left[\begin{array}{l}
E_{I}^{+} \\
E_{I}^{-}
\end{array}\right]=\left[\begin{array}{cc}
1-\frac{Z}{2 Z_{s} \cos \theta} & -\frac{Z}{2 Z_{s} \cos \theta} \\
\frac{Z}{2 Z_{s} \cos \theta} & 1+\frac{Z}{2 Z_{s} \cos \theta}
\end{array}\right]\left[\begin{array}{l}
E_{I I}^{+} \\
E_{I I}^{-}
\end{array}\right]
$$

with $Z_{s}=\frac{1}{\sigma d}$ the impedance of the sheet. This transfermatrix is calculated with the same material on both sides of the sheet. This is not a loss of generality since we can always multiply the sheet-transfermatrix with an interface-transfermatrix to account for the change in material parameters.

Finally we need a translation-transfermatrix to pass from one interface of a layer to its other interface. For a layer with thickness $d$, wavenumber $k=\omega \sqrt{\epsilon \mu}$ and angle of incidence $\theta$ in the layer this is given by

$$
T=\left[\begin{array}{cc}
e^{j d k \cos \theta} & 0 \\
0 & e^{-j d k \cos \theta}
\end{array}\right]
$$

This transfermatrix can cause numerical problems when $k$ is complex (lossy materials) or when $\theta$ is complex (evanescent waves). The reason is that the argument of one of the exponentials in these cases always will contain a positive real part. This could lead to numerical overflows and loss of precision in products of these matrices. However, for the applications here we never faced such problems. It would be different matter if integrations over plane wave spectra are performed involving the evanescent plane waves. This would be the case if Green functions for layered media are evaluated such as in [5]. In that case a recursive technique can be used to find the scattering of a stack of layers as outlined in [4]. This recursive technique however is more CPU-time consuming.

The transfermatrix method needs to be completed with backing equations at one side of the stack of layers. For a perfectly electric conducting backing we impose that $E^{+}=$ $-E^{-}$and for a free space backing that $E^{-}=0$.

The optimization in the continuous thickness space is defined as finding the vector of thicknesses $\bar{d}$ for which

$$
f(\bar{d})=\sup _{\left(f_{i}, \theta_{j}\right)}\left[\max \left(R_{T E}\left(f_{i}, \theta_{j}, \bar{d}\right), R_{T M}\left(f_{i}, \theta_{j}, \bar{d}\right)\right)\right]
$$

becomes minimal. $R_{T E}$ and $R_{T M}$ are the reflection coefficients for TE- and TM-waves, $\left(f_{i}, \theta_{j}\right)$ are the frequencies and angles of incidence. These reflection coefficients are calculated with the transfermatrix algorithm outlined above. In order to be able to find the global minimum of (4) the simulated annealing technique is used [3].

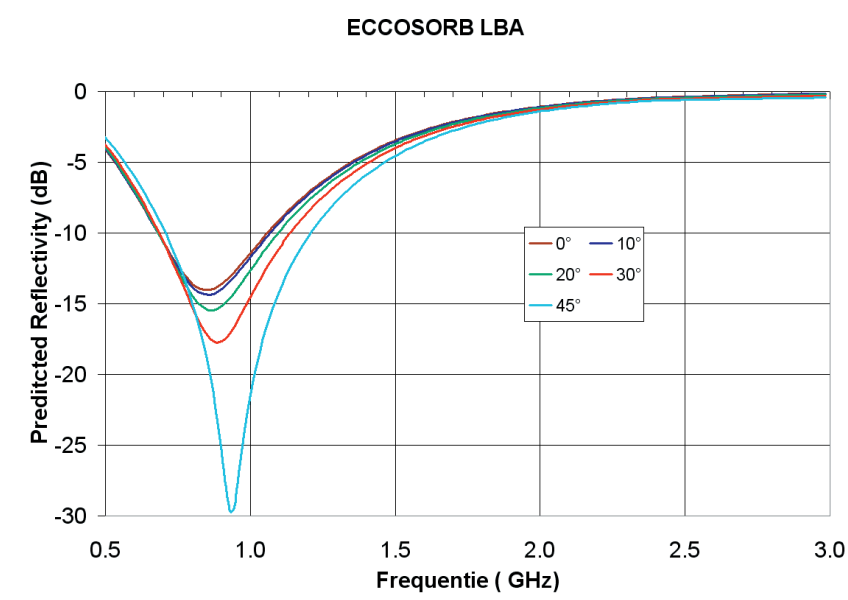

Fig. 1. Simulated reflectivity of the LBA absorber as a function of frequency for various angles of incidence.

ECCOSORB LBA: MEASURED COAX

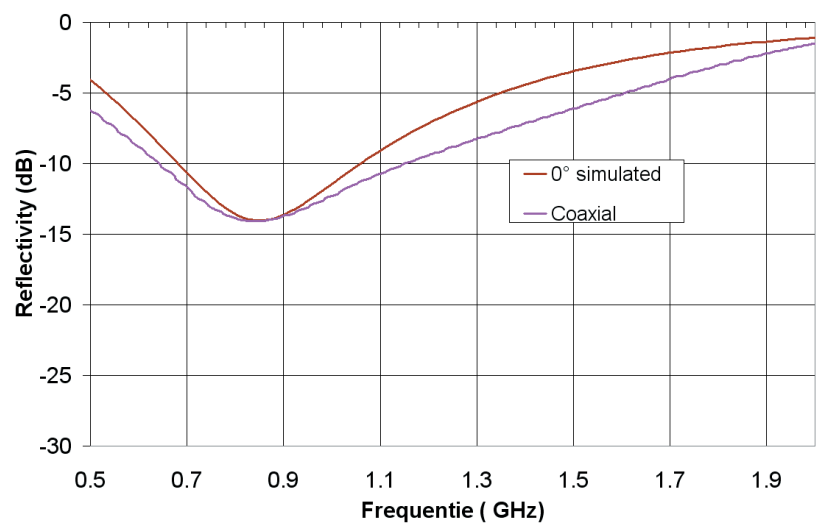

Fig. 2. Comparison between measurement in a coaxial line and simulation at perpendicular incidence for the LBA absorber. 
ECCOSORB LBA: MEASURED WAVEGUIDE

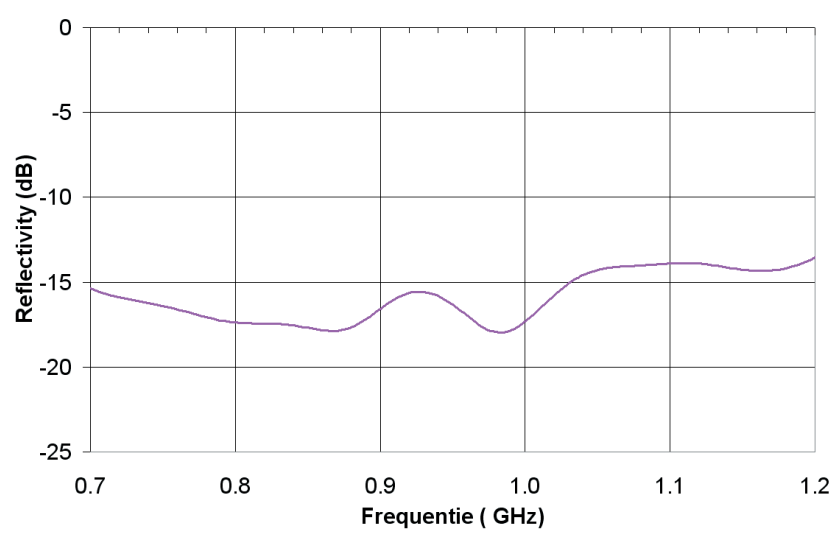

Fig. 3. Measurement in a rectangular waveguide for the LBA absorber.

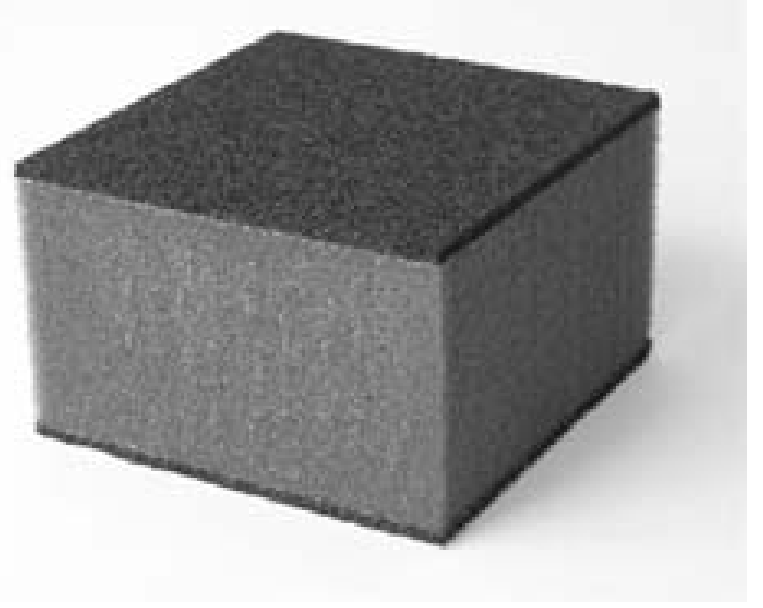

Fig. 4. Picture of the LBA absorber.

\section{REALIZATIONS}

\section{A. Loading Bay Absorber}

The first realization is a Loading Bay Absorber (ECCOSORB LBA). This absorber is used for RFID portals. The design had a total thickness constraint of $65 \mathrm{~mm}$. The best possible absorption performance was requested within an angle of incidence range from 0 to 45 degrees. The final design consisted of a symmetric design of three layers: a dielectric layer with thickness $4.3 \mathrm{~cm}$ sandwiched in between two absorbing layers with a thickness of $3.1 \mathrm{~mm}$. Fig. 1 shows the simulated reflection coefficients as a function of frequency for various angles of incidence. It is noted that the best performance is obtained for the highest angles of incidence. Better performance for low angles of incidence was not possible due to the total thickness constraint. Fig. 2 shows a comparison of the computed 0 degrees of incidence result with the measurement of a coaxial line and Fig. 3 shows measurements in a rectangular waveguide [6]. Measurements in a rectangular waveguide correspond to different angles of incidence at the same time. Note that the frequency range in Fig. 3 is limited. The agreement in Fig. 2 is excellent. Finally, Fig. 4 shows a picture of the LBA absorber.

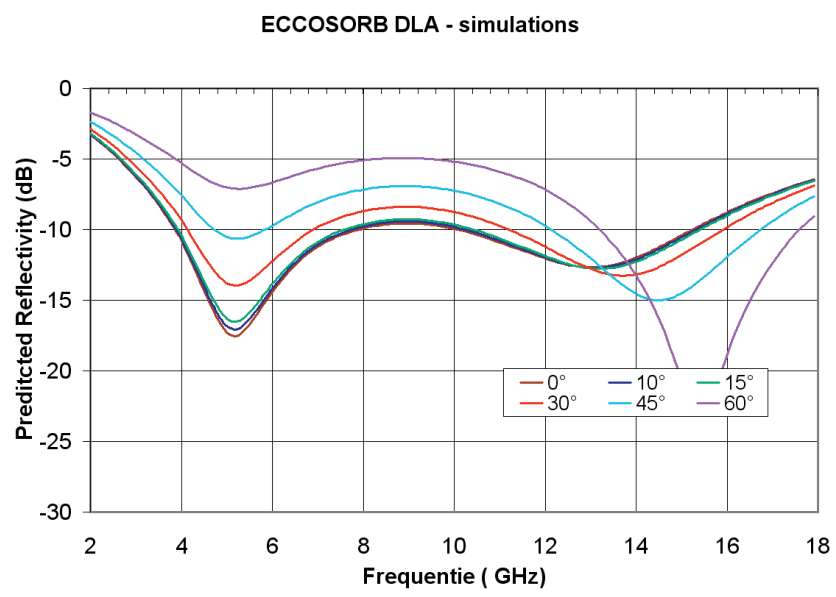

Fig. 5. Simulated reflectivity of the DLA absorber as a function of frequency for various angles of incidence.

ECCOSORB DLA: MEASURED NRL ARCH

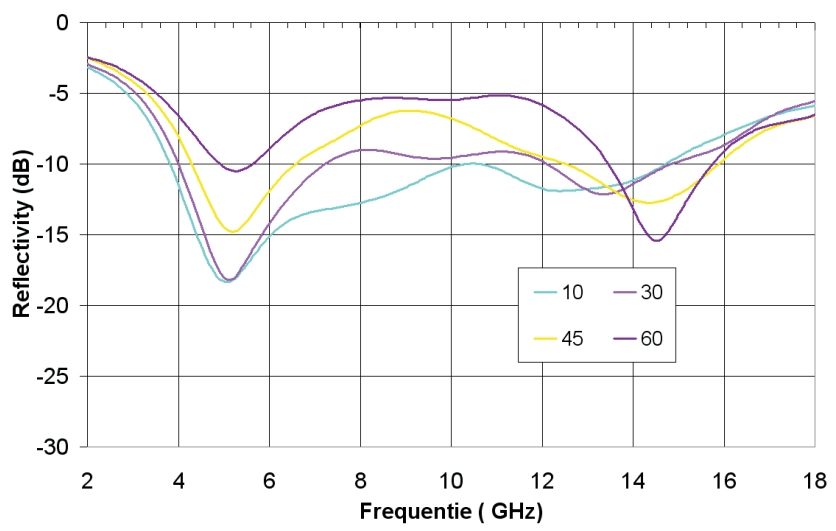

Fig. 6. Measurement of the reflectivity of the DLA absorber as a function of frequency for various angles of incidence.

\section{B. Dual Layer Absorber}

As a second realization we consider a Dual Layer Absorber (ECCOSORB DLA). The DLA absorber is suited for applications requiring reflectivity reduction at low frequencies in harsh environments, particularly on objects with contoured shapes. It consists of a fluorised-silicone matrix giving it excellent resistance against high temperatures and aggressive media. These severe restrictions pose limitations on the type of materials that can be selected from the portfolio. The DLA absorber is designed to reflect less than $-10 \mathrm{~dB}$ of normally incident energy relative to a metal plate between $4.5 \mathrm{GHz}$ and $15 \mathrm{GHz}$. The two layers have a thickness of respectively 1.05 $\mathrm{mm}$ and $4.35 \mathrm{~mm}$. Fig. 5 shows the simulated reflectivity as a function of frequency for various angles of incidence and Fig. 


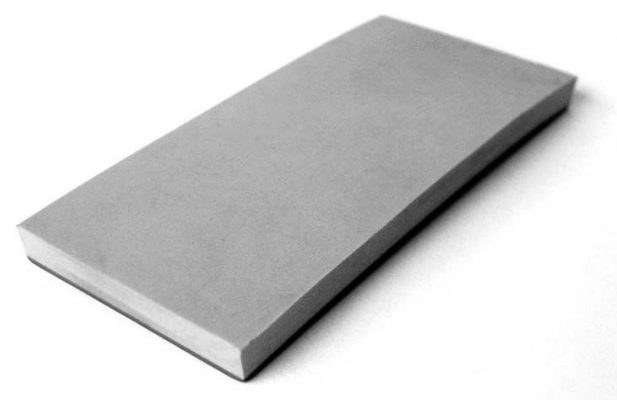

Fig. 7. Picture of the DLA absorber.

6 shows the corresponding measurement results on an NRLarch bipolar measurement system [7]. Due to the bipolar setup no measurements are available at low angles of incidence. The agreement between simulations and measurements is impressive. Finally Fig. 7 again shows a picture of the design.

\section{CONCLusions}

We presented an optimization scheme for the design of highperformance layered absorbers. The optimization consisted of an exhaustive search in a discrete portfolio space and a simulated annealing optimization in a continuous thickness space. The method was illustrated with two realizations.
Further research involves the inclusion of a sensitivity analysis in order to check the robustness of designs and in order to include measurement uncertainties on the material parameters of the used materials.

\section{ACKNOWLEDGMENT}

This work is supported by a research grant from the Institute for the Promotion of Innovation through Science and Technology in Flanders (IWT-Vlaanderen).

\section{REFERENCES}

[1] D.S. Wiele, E. Michielssen, and D.E. Goldberg, "Genetic algorithm design of Pareto optimal broadband microwave absorbers," IEEE Trans. on Electromagnetic Compatibility, Vol. 38, no. 3 , pp. 518-525, 1996.

[2] J.W. Bandler. W. Kellerman, and K. Madsen, "A superlinearly convergent minimax algorithm for microwave circuit design," IEEE Trans. on Microwave Theory and techniques, Vol. 33, no. 12, pp. 1519-1530, 1985.

[3] R.H.J.M. Otten, and L.P.P.P. van Ginneken, The Annealing Algorithm, Kluwer, Boston, 1989.

[4] F. Olyslager, B. Baekelandt, D. De Zutter, and M. Van Craenendonck, "Plane wave scattering at structures consisting of bianisotropic layers and resistive sheets," Proc. of the Joint 3rd International Conference on Electromagnetics in Aerospace Applications and 7th European Electromagnetic Structures Conference, Torino, Italy, pp. 295-298, 1993.

[5] N. Faché, F. Olyslager and D. De Zutter, Electromagnetic and Circuit Modelling of Multiconductor Transmission Lines, Oxford University Press, Oxford, United Kingdom, 1993.

[6] H. Pues, "Electromagnetic absorber measurement in a large waveguide", Proc. of the 8th Zurich Electromagnetic Compatibility Symposium, Zurich, Switserland, pp. 253-258, 1989.

[7] H. Pues, "Error-corrected wideband reflectivity measurement of microwave absorbing materials using the arch technique", Mikrowellen magazin, vol. 14, no. 6, pp. 524-525, 1988. 